\title{
AIDS E DIABETES MELLITUS VERSUS JUSTIÇA DISTRIBUTIVA NO SISTEMA PÚBLICO DE SAÚDE
}

Silvia Lucia de Castro Oliveira* Wilson Danilo Lunardi Filho**

\section{Resumo}

Reflexão sobre a forma de distribuição dos recursos públicos na área de saúde e as conseqüências decorrentes. Questiona-se a eqüidade da justiça distributiva. Propõe-se uma leitura contextual dos números oficiais, através de inter-relações que possibilitem a compreensão do significado das cifras governamentais. AIDS e Diabetes, escolhidas como paradigmas, representam, respectivamente, os grupos das Doenças Transmissíveis e das Não Transmissíveis. Os números oficiais relativos às duas doenças estão correlacionados em alguns aspectos pontuais: gastos com medicamentos prevalência, evolução de taxas de mortalidade. Alerta-se para as diferenças de atenção oficial marcantes entre as duas doenças e para as conseqüências sofridas por pessoas atingidas por Diabetes e por outras doenças não transmissíveis, decorrentes da insuficiência de recursos públicos destinados ao seu atendimento.

Descritores: justiça distributiva; doenças transmissíveis; doenças e agravos não transmissíveis; AIDS; Diabetes

\begin{abstract}
Reflection about the way of distributing public resources in the area of health and the ensuing consequences. The fairness of distributive justice is questioned. We propose a contextual reading of the official figures through interrelations that make it possible to understand the meaning of government figures. AIDS and Diabetes, which have been chosen as paradigms, represent, respectively, the groups of Transmitted Diseases and NonTransmitted Diseases. The official figures relating to the two diseases are correlated in some precise aspects such as expenses on drugs, prevalence, and evolution of mortality rates. We alert to the remarkable differences in official attention between these two diseases and to the consequences suffered by people affected with Diabetes and other nontransmitted diseases, arising from the insufficiency of public resources allotted for their health care.
\end{abstract}

Descriptors: fairness in the distribution and use of resources; transmitted diseases; diabetes

Title: AIDS and diabetes mellitus versus distributive justice in the public health system

\section{Resumen}

Reflexión sobre la forma de distribución de los recursos públicos en el área de salud y las consecuencias provenientes. Se cuestiona la equidad de la justicia distributiva. Se propone una lectura contextual de los números oficiales, a través de interrelaciones que posibiliten la comprensión del significado de las cifras gubernamentales. SIDA y Diabetes, escogidas como paradigmas, representan, respectivamente, los grupos de las Enfermedades Transmisibles y de las No Transmisibles. Los números oficiales relativos a dos enfermedades están correlacionados en algunos aspectos puntuales: gastos con medicamentos, prevalencia, evolución de tasas de mortalidad. Se alerta para las diferencias de atención oficial fuertes entre las dos enfermedades y para las consecuencias sufridas por personas acometidas por Diabetes y por otras enfermedades no transmisibles, provenientes de la insuficiencia de recursos públicos destinados a su atención. Descriptores: equidad en la distribución y uso de los recursos; enfermedades transmisibles; diabetes

Título: SIDA y Diabetes Mellitus versus Justicia Distributiva en el Sistema Público de Salud

\section{Introdução}

Quando se correlacionam doenças crônicas e o sistema público de saúde, a primeira idéia que circula, normalmente, é associada à escassez e a solução parece estar ligada diretamente ao oferecimento de mais recursos. Essa insuficiência é, de fato, muitas vezes, notória na avaliação das ações, porém, há um outro aspecto, raramente comentado: a equidade na distribuição dos recursos existentes.

Esta reflexão remete à ética da distribuição de recursos. A justiça distributiva impõe que, "se algo deve ser distribuído, que a distribuição não seja arbitrária, ela tem que ser justa"(1:140). Em outras palavras, requer certa igualdade de tratamento, embora não haja uma forma universal de atribuí-la. Por certo, depende das circunstâncias, mas, para ser justa, a repartição deve garantir determinada proporcionalidade.

\section{2 Ética e eqüidade na distribuição de recursos}

Em termos de justiça formal, a referência maior é a Constituição Federal do Brasil(2), que assegura como um dos direitos sociais básicos a saúde. O art. 196 - do Capítulo relativo à Saúde é específico, quando traz que "a saúde é direito de todos e dever do Estado, garantido mediante políticas sociais e econômicas que visem à redução do risco de doença e de outros agravos e ao acesso universal e igualitário às ações e serviços para sua promoção, proteção e recuperação (grifos nossos).

Analisar a distribuição dos recursos públicos em saúde é assunto complexo. Os dados oficiais são apresentados de forma fragmentada, por relatórios de ações isoladas.
Normalmente, não estão atualizados. Por este motivo, ao buscarem-se referências para este trabalho, obtivemos apenas algumas informações pontuais, extraídas de fontes oficiais (notícias divulgadas pelo governo, em sites, revistas ou jornais), comprovando que os números apresentados soltos de referências perdem-se e são puro dado, num vácuo desligado do contexto de seu endereço histórico e social. Os fragmentos da contabilidade oficial servem apenas para vislumbrar a realidade, mas compreendê-la exige um exercício de atenção e análise crítica.

Num primeiro olhar, os dados impressionam pela grandeza: cifras milionárias ou até mesmo bilionárias ilustram as ações estatais. Números que não fazem parte da realidade da vida cotidiana, valores que ultrapassam o imaginário e dificultam a sua visualização. Raramente, é feita uma mínima correlação na análise dos números apresentados.

Assim, é preciso decifrar, ou "des-cifrar", desconstituir as cifras, resgatando dos números a humanidade aos quais se referem. A escrita enigmática da contabilidade pública pode ser melhor avaliada ao estabelecerem-se parâmetros que balizem a compreensão. É, neste sentido, que alinhavamos, aqui, algumas idéias.

\section{Des-cifrando os números da AIDS e do Diabetes}

Buscando estabelecer algumas conexões, nossa proposta é a de refletir sobre ações públicas que têm sido desenvolvidas em relação a duas grandes epidemias: a Síndrome da Imunodeficiência Humana Adquirida (AIDS) e Diabetes mellitus. Esta escolha não foi casual ou aleatória. As

\footnotetext{
* Psicóloga Clínica. Psicóloga da Secretaria Municipal de Saúde da Cidade do Rio Grande/RS. Mestre em Enfermagem, Membro do Núcleo de Estudos e Pesquisas em Saúde - NEPES/FURG. **Enfermeiro. Professor Adjunto IV do Departamento de Enfermagem da Fundação Universidade Federal do Rio Grande. Doutor em Enfermagem. Membro do Núcleo de Estudos e Pesquisas em Saúde - NEPES/FURG. Orientador.

E-mail do autor: lunardifilho@terra.com.br
} 
duas doenças estão relacionadas à mudança do perfil epidemiológico do Brasil, ocorrida nas últimas quatro décadas do século $X X$, em que as doenças infecciosas transmissíveis tiveram uma progressiva queda na morbimortalidade, enquanto que as Doenças e Agravos Não Transmissíveis (DANTs) sofreram elevação acentuada, conforme estudos da Fundação Nacional de Saúde (FUNASA), publicados em seu site oficial ${ }^{(30}$. Essa "transição epidemiológica", como a mudança é denominada, provocou repercussões que necessitam ser consideradas no planejamento das ações de saúde.

Conforme dados do Ministério da Saúde ${ }^{(4)}$, o número de casos de AIDS no Brasil é de 277.141 pessoas (de 1980 a setembro de 2003). Cerca de $50 \%$ dessas pessoas já foram a óbito. A estimativa, em 2000, era de que 600 mil pessoas estariam vivendo com o vírus da imunodeficiência humana (HIV) no Brasil, liderando o ranking da epidemia em número absoluto de casos. Entretanto, na comparação do número de casos pelo número de habitantes, o Brasil situava-se na $14^{a}$ posição, com $0,57 \%$ da população adulta contaminada.

Em relação ao Diabetes, segundo dados da Organização Mundial de Saúde (OMS), 5 milhões de brasileiros têm a doença, o que coloca o País em $6^{\circ}$ lugar no mundo em casos dessa doença, mas esse número pode ser ainda maior, o que demonstra a sua magnitude e importância. A OMS também divulga a previsão de que, em 2010, o número de pessoas com Diabetes chegue a 11 milhões. Somente com a realização do Estudo Multicêntrico sobre Prevalência de Diabetes Mellitus no Brasil $^{5}$, realizado no final da década de 80 , em nove capitais brasileiras, passou a ser conhecida a sua prevalência.

Foi constatado, então, que o coeficiente de prevalência no Brasil na população urbana de 30 a 69 anos, era de 7,6\%, magnitude semelhante a de países desenvolvidos. Em relação ao sexo, a prevalência do Diabetes é semelhante para homens e mulheres, aumentando consideravelmente com o progredir da idade. Dados brasileiros mostram que a prevalência varia de $2,7 \%$ para o grupo etário de 30 a 39 anos, até $17,4 \%$ para 0 grupo etário de 60 a 69 anos $^{(5)}$.

A partir de 1996, o governo começou a distribuir gratuitamente a terapia anti-retroviral de alta potência (High Active Antiretroviral Therapy). Conhecida popularmente como "coquetel", a terapia é o esquema terapêutico composto por 2 Inibidores da Transcriptase Reversa - ITR (zidovudina - AZT) e 1 Inibidor de Protease, que foi apresentado pelo Dr. David Ho, em 1996, na Conferência Mundial de AIDS em Vancouver. A partir da sua distribuição, o crescimento da epidemia estabilizou-se, numa média de 20 mil novos casos por ano, até 1999. Em 2000, houve indício de declínio, com o registro de 15 mil novos casos, com a estimativa da existência de 600 mil pessoas entre 15 e 49 anos vivendo com o HIV (coeficiente de prevalência de 0,65\%)(6).

No primeiro semestre de 2001, confirmou-se a queda, embora os números só possam ser considerados definitivos, após três anos de notificação. Atualmente, cerca de 100 mil brasileiros recebem o tratamento gratuitamente. O decréscimo agudo da letalidade é um dos indicadores mais efetivos da eficácia das ações de tratamento desenvolvidas pelo governo brasileiro. No Brasil, a mortalidade por AIDS teve uma queda de $50 \%$ e a qualidade de vida das pessoas portadoras do HIV alcançou uma melhora significativa, como sinal da eficácia da política de distribuição gratuita e universal dos anti-retrovirais ${ }^{(7)}$.

Em relação ao Diabetes, a evolução foi inversa, tendo sido registrada acentuada progressão na mortalidade: em 1998, era a $7^{a}$ causa de morte e, em 1999, passou a ser a $6^{a}$. Anualmente, mais de 25 mil pessoas morrem devido a esta doença. A situação ainda é agravada pelo fato de que, segundo estatísticas do Ministério da Saúde, $50 \%$ das pessoas não sabem que têm diabetes e $23 \%$ conhecem seu diagnóstico, mas não fazem qualquer tipo de tratamento(6). Em relação às principais causas de mortalidade no Estado do Rio Grande do Sul, estão as doenças isquêmicas coronarianas, cérebro- vasculares e hipertensão arterial. É importante observar que estas e outras doenças, por vezes, estão associadas ao Diabetes, embora no registro de óbito conste apenas o nome de uma delas como a causa imediata(8).

\section{Recursos aplicados no tratamento da AIDS e do diabetes}

No ano de 2000, a Coordenação Nacional de DST/AIDS, do Ministério da Saúde, informou ter gasto 1 bilhão de reais no tratamento e prevenção do HIVIAIDS. O compromisso do governo brasileiro em relação à AIDS destaca-se em nível internacional, tendo chegado a destinar $\mathrm{R} \$ 2,5$ milhões para financiar projetos de prevenção à AIDS e tratamento de portadores do HIV em países da África, Ásia, América Latina e do Caribe.

O destaque da ação brasileira pode também ser avaliado diante do fato de que, em 2002, $90 \%$ das 40 milhões de pessoas vivendo com HIV e AIDS viviam em países em desenvolvimento e apenas 250 mil pessoas tinham acesso gratuito aos medicamentos anti-retrovirais. No Brasil, os pacientes com HIV e AIDS recebem todo o tratamento de que necessitam, havendo cerca de 100 mil brasileiros que utilizam antiretrovirais ${ }^{(7)}$.

A prioridade do custeio das despesas com DST/AIDS é noticiada pelo Ministério da Saúde, que informa que, no ano de 1998, seus gastos totais com saúde sofreram um decréscimo de cerca de $1 \%$, enquanto os gastos com DST/AIDS apresentaram um crescimento real de $28,3 \%$, comprometendo de $2,0 \%$ a $2,6 \%$ do total gasto nos anos de 1997 e 1998, respectivamente ${ }^{(7)}$

A política de prioridade do atendimento aos doentes de AIDS, estabelecida por Lei Federal, em 1996, que garantiu a medicação necessária a essas pessoas, tem determinado que mais de $25 \%$ dos gastos do Ministério da Saúde destinados à aquisição centralizada de medicamentos venham sendo absorvidos com medicamentos para a AIDS, desde $1997^{(7)}$

A maior parcela dos gastos foi destinada ao tratamento: $73 \%$ (U\$ 247,7 milhões), em 1997, e 80,8\% (U\$ 352,3 milhões), em 1998. Os gastos com prevenção representaram 13,2\% e 9,6\% do aplicado, respectivamente, em 1997 e 1998, enquanto os gastos com o desenvolvimento institucional corresponderam a $12,4 \%$ e 9,3\%. Por seu turno, os dispêndios com Vigilância Epidemiológica representaram 1,4\% dos gastos, em 1997, e apenas 0,3\%, em 1998.

Os gastos nacionais com DST/AIDS, incluindo estimativas sobre a participação de estados, municípios, universidades, outros órgãos governamentais, empresas e famílias, alcançaram o montante de U\$ 634,3 milhões, em 1998, ou seja, o equivalente a $0,07 \%$ do PIB e um per capita de U\$ $4,05^{(8)}$. Este cálculo per capita, apresentado pela burocracia oficial, considera o montante gasto em relação ao total da população brasileira, o que é um equívoco matemático. É preciso atentar que estas despesas, especialmente as de natureza curativa, que representam o maior gasto, destinaramse a um universo inferior a 80 mil pessoas que, naquele ano, utilizavam anti-retrovirais.

Buscando o significado dos números, na matemática humana que aqui propomos, verificamos que, em 1998, o valor gasto em nível federal, exclusivamente com o tratamento das pessoas com HIVIAIDS, foi de U\$359.270.000,00 que, dividos por 80 mil pessoas que utilizaram o tratamento naquele ano, resulta em U\$ 4.490,00 per capita ${ }^{(9)}$. Para transformar o valor em moeda corrente nacional, que baliza o cotidiano, convertemos esse valor pela cotação do dólar de 11 de junho de 2003 , $(R \$ 2,853)^{(10)}$, o que resulta no gasto de $R \$ 12.825,93$ per capita/ano.

Os expressivos gastos com AIDS estão diretamente relacionados com os bons resultados obtidos, como referido no Relatório de Pesquisa de Pio Marins e colaboradores ${ }^{(11)}$. Este relatório apresenta que as possíveis diferenças existentes na sobrevivência causadas por diferença de sexo, situação 
socioeconômica, idade e categorias de exposição foram anuladas, quando foi garantido o mesmo acesso ao tratamento a todos. Textualmente, o relatório conclui que

esse fato (o acesso ao tratamento anti-retroviral) é de grande importância, pois demonstra que, independente dos fatores socioeconômicos e culturais, o uso da terapêutica muda a história natural da doença e proporciona maior eqüidade na sobrevivência dos afetados $^{(11: 44)}$ (grifos nossos).

Também é de salientar-se que esse fato traz uma outra evidência de grande relevância para o nosso País e para outros países em desenvolvimento, pois a maior parte dos nossos pacientes tem baixo nível de escolaridade e baixo nível socioeconômico. Os resultados ora apresentados demonstram que essas

condições inadequadas vividas por pessoas afetadas pelo vírus não são impedimentos para que se beneficiem do tratamento em iguais condições àqueles que vivem de maneira mais favorecida $^{(11: 44)}$ (grifos nossos).

Em relação ao Diabetes, a situação é exatamente oposta. O Diabetes é uma doença que, hoje, tem recursos científicos e tecnológicos sofisticados para o seu tratamento, mas esses avanços não estão democratizados pelo Sistema único de Saúde (SUS). Os pacientes do SUS são tratados como se fossem todos iguais: recebem um kit básico de seringas (muitos municípios nem sequer as distribuem) e insulina NPH (mista ou humana, dependendo unicamente de escolhas burocráticas). O monitoramento glicêmico é disponibilizado descontinuamente nos postos de saúde, não sendo oferecidos recursos para o controle domiciliar.

Todos estes aspectos colaboram para o surgimento de agravos, pois a condição de pobreza contribui, negativamente, para a evolução da doença, como refere a Declaração das Américas Sobre Diabetes (DOTA) ${ }^{(12)}$, ratificada em julho de 1996. Este é um documento firmado por vários países das Américas, entre os quais o Brasil, que descreve um plano estratégico sobre Diabetes para as Américas e representa também a coalizão das organizações que lutam pela implementação dos objetivos enunciados.

Esta declaração registra que o Diabetes, especialmente quando inadequadamente controlado, pode representar um pesado encargo econômico para o indivíduo e a sociedade. A maior parte dos custos diretos do Diabetes relaciona-se com as suas complicações que, muitas vezes, podem ser reduzidas, retardadas ou, em certos casos, evitadas. Dependendo do país, as estimativas disponíveis indicam que o Diabetes pode gerar de $5 \%$ a $14 \%$ das despesas de atenção à saúde. A pobreza exerce efeito negativo sobre a atenção ao Diabetes, restringindo a probabilidade de ser diagnosticado corretamente, a qualidade da educação recebida, a adequação da atenção, a capacidade de pagar o tratamento e agravando o risco de manifestar complicações graves.

A Campanha Nacional de Detecção de Suspeitos de Diabetes, executada pelo Ministério da Saúde em março de 2001, realizou testes de glicemia capilar em 20,7 milhões de pessoas e identificou 3,2 milhões de casos suspeitos. Para a campanha, o ministério gastou $\mathrm{R} \$ 31$ milhões, na compra de 30 mil glicosímetros (medidores dos índices de glicose) e R 1 milhão, na capacitação de profissionais de saúde para fazerem os exames(12). Buscando-se estabelecer paradigmas matemáticos, dividimos o total do valor aplicado (R $\$ 32$ milhões) pelo número de pessoas que realizaram os exames ( 20,7 milhões). Verificamos que a campanha de Diabetes teve um custo de R\$ 1,54 por pessoa (teste). A campanha detectou $15,45 \%$ de pessoas testadas suspeitas de diabetes.

Já com relação à hipertensão, condição que muitas vezes está combinada com o Diabetes, o Ministério da Saúde realizou, em novembro de 2001, a Campanha Nacional de Detecção de Hipertensão, quando foram feitos 11 milhões de exames e identificados 4 milhões de suspeitos (36,3\%). O Ministério comprou 34,5 mil tensiômetros (aparelhos usados para medir a pressão arterial) ao custo total de $\mathrm{R} \$ 2$ milhões. O valor investido corresponde a $\mathbf{R} \mathbf{\$} \mathbf{0 , 1 8}$ por pessoa/exame, observando-se que os tensiômetros são equipamentos e que continuarão a ser utilizados, reduzindo ainda mais sua relação custo-benefício ${ }^{(12)}$.

"Além da prevenção, o Ministério investe forte no tratamento, distribuindo gratuitamente os medicamentos necessários aos pacientes" (grifos nossos), diz a nota oficial.

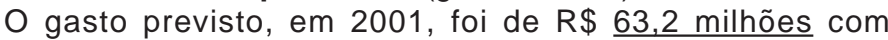
medicamentos para Diabetes e $\underline{R} \$ 100$ milhões com remédios para Hipertensão. O valor previsto para o gasto anual $(R \$ 63,2$ milhões) dilui-se, quando analisado em relação ao número de pessoas com Diabetes estimado pela OMS (5 milhões). Verificamos que o gasto previsto em medicamentos por pessoa correspondeu a R\$12,64 anuais ou R \$ 1,05 mensais. Ainda que se considere nessa estimativa que 50\% desconhecem a doença e que aproximadamente $25 \%$ dos que sabem seu diagnóstico não se tratam, restariam 1.875 .800 de pessoas para as quais foram destinados os $\mathrm{R} \$ 63,2$ milhões em medicamentos: o investimento ficaria em $\mathrm{R} \$ 33,69$ per capital ano ou $\mathrm{R} \$ 2,80$ mensais.

Os números oficiais apresentados pelo Ministério da Saúde indicam a situação registrada na DOTA ${ }^{(13)}$, de que milhões de pessoas com Diabetes não recebem tratamento adequado. Devido ao inadequado controle, correm um risco consideravelmente maior de complicações cardíacas, derrame cerebral, cegueira, insuficiência renal, amputação de membros inferiores e morte prematura. Vale referir que apenas $30 \%$ dos Ministérios de Saúde do mundo destinam verbas para a redução de DANTs. Para o representante do Brasil na Organização PanAmericana de Saúde (OPAS), o Brasil tem o melhor programa de prevenção de Hipertensão e Diabetes do mundo ${ }^{13}$.

\section{Reflexões necessárias sobre a justiça distributiva}

Ao trazer-se dados da situação da AIDS e do Diabetes, ao comparar-se a evolução dessas epidemias e os recursos aplicados no tratamento de cada uma delas, constata-se uma inversão na proporcionalidade. AIDS é a única doença que tem recebido a atenção que garante o artigo 196 da Constituição: todos os pacientes, de modo igualitário, têm acesso de forma gratuita aos tratamentos que necessitem para manter sua saúde, independentemente de seus custos. Houve um enfrentamento das iniqüidades e foi garantida a universalidade e os direitos constitucionais aos cidadãos que têm AIDS.

Cabe novamente trazer que a Constituição Federal é para todos os cidadãos, sem discriminações ou preferências. É a lei magna, superior a todas as demais, mas a realidade mostra que outros dispositivos inferiores podem funcionar de modo mais imperativo - como é o caso da Lei 9.313, de 13 de novembro de $1993^{(14)}$, que garante que os pacientes de AIDS do País tenham acesso a todos os avanços terapêuticos disponíveis no mercado mundial, a despeito de seus altos custos $^{(11: 38)}$.

Quando determinados grupos organizados obtêm a garantia do cumprimento de seus direitos constitucionais e tais direitos, no seu cumprimento, ficam restritos apenas a estes grupos organizados, a justiça obtida é individualista. "A ética individualista é conservadora das desigualdades sociais"(1:151).

A ação governamental desenvolvida em relação à AIDS tem sido exemplar, mas, infelizmente, esgota-se em si mesma. Por este fato, tem tornado os pacientes com HIVIAIDS privilegiados em relação aos demais pacientes. Não que haja privilégio na doença, mas no atendimento a ela oferecido, em detrimento de todas as demais doenças.

Os números aqui apresentados comprovam, também, que tem havido insuficiente distribuição de recursos para as 
doenças não transmissíveis, como é o caso do Diabetes, que tem sido negligenciada nas ações governamentais. A silenciosa discriminação dos pacientes com DANTs - vítimas da injustiça distributiva, tem suas conseqüências não somente no aumento dos custos em atendimentos de alta-complexidade (amputações, hemodiálises, internações hospitalares), mas, principalmente, no sacrifício de muitas vidas humanas.

Um exemplo recente reforça a comprovação dessa negligência: no dia 26 de junho de 2003, o governo emitiu a Medida Provisória (MP) 123, publicada no Diário Oficial da União de 27/06/2003, visando a redução do preço de medicamentos. Nesta MP, o governo esqueceu de incluir na lista os medicamentos anti-hipertensivos e remédios para Diabetes, além de muitos outros essenciais ${ }^{(15)}$.

A matemática pública parece desconhecer o drama humano gerado pela injusta distribuição dos recursos. O potencial humano sacrificado pelo precoce afastamento do trabalho, as vidas ceifadas por doenças evitáveis, os destinos de pacientes e dos familiares não entram nas contas do governo. A burocracia desconhece essas minúcias, ocupada com o funcionamento da grande máquina estatal. A leitura atenta dos dados oficiais comprova a situação trágica vivenciada por usuários e presenciada pelos profissionais do sistema público de saúde: algumas patologias, principalmente da categoria das doenças não transmissíveis, têm sido preteridas em relação a outras.

\section{Referências}

1. Nedel J. Ética, direito e justiça. Porto Alegre (RS): Edipucrs; 2000. $248 p$.

2. Senado Federal (BR). Constituição: República Federativa do Brasil: 1988. Brasília (DF): Centro Gráfico; 1988. 292 p.

3. FUNASA - Fundação Nacional da Saúde - Guia de Vigilância Epidemiológica. Disponível em: URL: <http://www.funasa.gov.br/pub/ pub00.htm\#>. Acessado em: 23 out 2003.

4. Ministério da Saúde (BR). Coordenação Nacional DTS/AIDS. Disponível em: URL: <http:// www.aids.gov.br/>. Acessado em: 11 out 2003.

5. Ministério da Saúde (BR). Programa brasileiro de hipertensão e diabetes. Disponível em: URL: <http//portalweb01.saude.gov.br/ saude/buscas.cfm?inicio=16>. Acessado em: 23 out 2003.

6. Pace AE, Foss MC, Ochoa-Vigo K, Hayashida M. Fatores de risco para complicações de extremidades inferiores de pessoas com diabetes mellitus. Revista Brasileira de Enfermagem, Brasília (DF) 2002 set/out; 55(5): 514-21.

7. Boletim Epidemiológico AIDS - Ano XV n.01 - outubro de 2001 a março 2002. Disponível em: URL:<http://www.aids22.hpg.ig.com.br/ ciência educaçao/82/index pinrl.html>. Acessado em: 11 mar 2003.

8. Davidson SL, Gotlieb E, Castilho A, Buchalla CM. Impacto da aids na esperança de vida, Brasil, 2000 Boletim Epidemiológico - abril a dezembro 2002. Disponível em URL: <http://www.aids.gov.br>. Acessado em: 08 set 2003.

9. Cotação do Dólar. Jornal do Comércio, Porto Alegre (RS) 2003 jun 12; capa.

10. Marins JRP, Jamalw LF, Chen S, Hudes ES, Barbosa Jr A, Barros MBA. Sobrevivência atual dos pacientes com aids no Brasil: evidência dos resultados de um esforço nacional. Relatório de pesquisa. Boletim Epidemiológico Aids 2002 out/mar;15(1):37-45.

11. Campanha Nacional de Detecção de Suspeitos de Diabetes - março de 2001. Disponível em URL: <http://www.dota.org/default_s.asp>. Acessado em: 08 set 2003.

Data de Recebimento: 08/05/2004

Data de Aprovação: 22/12/2004 